\title{
RELAÇÕES SOCIAIS E DIMENSÕES ÍNTIMAS DE IDOSOS AFETADOS POR HANSENÍASE*
}

Lucian da Silva Viana1, Maria Isis Freire de Aguiar², Ítalo Rodolfo Silva ${ }^{3}$, Nair Portela Silva Coutinho ${ }^{4}$, Dorlene Maria Cardoso de Aquino ${ }^{5}$

${ }^{1}$ Enfermeiro. Discente da Residência Multiprofissional em Oncologia pelo Instituto Nacional de Câncer e da Especialização em Estomaterapia pela Universidade do Estado do Rio de Janeiro. Rio de Janeiro, RJ, Brasil.

${ }^{2}$ Enfermeira. Doutora em Enfermagem. Docente do Departamento de Enfermagem da Universidade Federal do Ceará. Fortaleza, CE, Brasil.

${ }^{3}$ Enfermeiro. Mestre em Enfermagem. Docente do Departamento de Enfermagem e Obstetrícia da Universidade Federal do Rio de Janeiro. Macaé, RJ, Brasil.

${ }^{4}$ Enfermeira. Doutora em Ciências da Saúde. Docente do Departamento de Enfermagem da Universidade Federal do Maranhão. São Luís, MA, Brasil.

${ }^{5}$ Enfermeira. Doutora em Patologia Humana. Docente do Departamento de Enfermagem da Universidade Federal do Maranhão. São Luís, MA, Brasil.

RESUMO: Objetivou-se avaliar relações sociais e íntimas de pessoas idosas com hanseníase. Abordagem quantitativa, com 60 idosos em dois Programas de Controle da Hanseníase, em uma capital do nordeste brasileiro, utilizando o Domínio das Relações Sociais do World Health Organization Quality of Life - brefe a faceta Intimidade do World Health Organization Quality of Life - older adults, com análise estatística descritiva. A coleta de dados ocorreu entre dezembro de 2012 e junho de 2013. No Domínio das Relações Sociais, a satisfação foi de $85 \%$ nas relações pessoais, $78,3 \%$ no suporte social e $60 \%$ em atividade sexual. Na Faceta Intimidade, os idosos apresentaram menor satisfação. As Relações Sociais dos idosos com hanseníase apresentou alto escore devido à rede de apoio social, trazendo expressiva satisfação e se refletindo na sua qualidade de vida. Conclui-se sobre a importância da Enfermagem articular estratégias de práticas educativas e de cuidados à pessoa idosa e com hanseníase.

DESCRITORES: Relações interpessoais; Idoso; Enfermagem; Hanseníase.

\section{SOCIAL RELATIONSHIPS AND INTIMATE DIMENSIONS OF ELDERLY INDIVIDUALS AFFECTED BY HANSEN'S DISEASE}

ABSTRACT: The aim of this study was to assess social and intimate relationships of elderly individuals with Hansen's disease. This study has a quantitative approach, with 60 elderly individuals from two Hansen's Disease Control Programs in the capital city of a Brazilian Northeastern state, using the Social Relationships domain of the World Health Organization Quality of Life - bref and the Intimacy facet of the World Health Organization Quality of Life older adults, with descriptive statistical analysis. Data were collected between December 2012 and June 2013. In the Social Relationships domain, satisfaction was found at $85 \%$ in personal relationships, $78.3 \%$ in social support and $60 \%$ in sexual activity. The elderly individuals presented lower satisfaction in the Intimacy facet. The Social Relationships of elderly individuals with Hansen's disease presented a high score due to the social support network, which presented significant satisfaction and reflected on quality of life. In conclusion, it is important that nursing coordinates strategies for educational and care practices for elderly individuals affected by Hansen's disease.

DESCRIPTORS: Interpersonal relations; Aged; Nursing; Leprosy.

\section{RELACIONES SOCIALES Y DIMENSIONES ÍNTIMAS DE ANCIANOS ATINGIDOS POR ENFERMEDAD DE HANSEN}

RESUMEN: El objetivo fue evaluar relaciones sociales así como íntimas de personas ancianas con enfermedad de Hansen. Abordaje cuantitativo, con 60 ancianos en dos Programas de Control de la enfermedad de Hansen, en una capital del nordeste brasileño, utilizando el Dominio de las Relaciones Sociales del World Health Organization Quality of Life - brefy la categoría Intimidad del World Health Organization Quality of Life - older adults, con análisis estadístico descriptivo. Los datos fueron obtenidos entre diciembre de 2012 y junio de 2013. En el Dominio de las Relaciones Sociales, la satisfacción fue de $85 \%$ en las relaciones personales, $78,3 \%$ en el apoyo social y $60 \%$ en actividad sexual. En la categoría Intimidad, los ancianos presentaron menor satisfacción. Las Relaciones Sociales de los ancianos con enfermedad de Hansen presentó alto escore a causa de la red de apoyo social, trayendo expresiva satisfacción y reflejando en su cualidad de vida. Se concluye que es muy importante que la Enfermería articule estrategias de prácticas educativas y de cuidados al anciano con enfermedad de Hansen.

DESCRIPTORES: Relaciones interpersonales; Anciano; Enfermería; Enfermedad de Hansen.

*Artigo extraído de monografia intitulada: "Qualidade de vida de idosos frente a condição de envelhecer afetados por hanseníase". Universidade Federal do Maranhão, 2013.

\section{Autor Correspondente:}

Lucian da Silva Viana

Universidade do Estado do Rio de Janeiro

R. Carlos de Carvalho, 47 - 20230-180 - Rio de Janeiro, RJ, Brasil

E-mail: lucianviana@yahoo.com.br
Recebido: 31/05/2015 Finalizado: 04/08/2015 


\section{INTRODUÇÃO}

O processo de envelhecimento se constitui em fenômeno complexo que permeia pluralidades contextuais concernentes aos fatores culturais, econômicos, políticos, sociais e, ao mesmo tempo, especificidades que se particularizam à pessoa idosa nas dimensões psicossociais, biológicas e espirituais ${ }^{(1-3)}$. Nesse sentido, pensar e favorecer a saúde da pessoa idosa requer a compreensão do envelhecimento em sua multidimensionalidade. Logo, este fenômeno se constitui em desafio transdisciplinar ${ }^{(3)}$.

A Organização Mundial da Saúde (OMS) classifica como idoso a pessoa com mais de 65 anos de idade em países desenvolvidos e, com mais de 60 anos de idade em países em desenvolvimento ${ }^{(4)}$, tal qual o Brasil. Para a Organização PanAmericana de Saúde, o envelhecimento consiste em processo sequencial, individual, acumulativo, irreversível, universal, natural ${ }^{(5)}$. Todavia, cumpre destacar que o processo de envelhecimento, como sistema dinâmico, é influenciado por fatores intervenientes, conforme mencionando anteriormente ${ }^{(1-3)}$, dentre os quais estão as condições crônicas de saúde e seus impactos nas interações sociais/afetivas e dimensões íntimas da pessoa idosa ${ }^{(1)}$.

As condições crônicas de saúde, em suas vertentes multifacetadas, potencializam na pessoa idosa o comprometimento da capacidade funcional ${ }^{(3,6-7)}$, refletindo no grau de dependência para a realização do autocuidado; na sobrecarga de cuidadores, especialmente ao cuidador principal ${ }^{(6)}$; na dinâmica e funcionalidade familiar, entre outros. Sendo assim, representa conformação ampla do processo de saúdedoença.

No âmbito das condições crônicas de saúde, está a hanseníase - uma doença infectocontagiosa de caráter crônico, causada pelo Mycobacterium leprae, que possui predileção para a pele e nervos periféricos. Apresenta-se como uma das doenças mais antigas da humanidade e, apesar de, desde 1986, já existir a cura por meio da poliquimioterapia, ainda constitui importante problema de saúde pública no Brasil(8), onde representa o primeiro lugar na incidência e o segundo lugar na prevalência mundial, perdendo somente para Índia. No âmbito das Américas, é responsável por mais de $90 \%$ do número de casos registrados ${ }^{(9)}$.

O fenômeno em pauta possui como agravante a concepção estereotipada da hanseníase como uma doença permeada por tabus, medos e preconceitos que, arraigados aos fatores históricos, culturais e ao déficit de conhecimento, estigmatizam o doente de modo a influenciar sua capacidade para a interação social, refletindo, consequentemente, no seu isolamento ${ }^{(10)}$.

Assim sendo, torna-se fundamental conheceros aspectos intervenientes à capacidade de interação social das pessoas idosas com hanseníase, uma vez que, para planejar e implementar estratégias de intervenção faz-se necessário identificar as nuances que desencadeiam e/ou potencializam o fenômeno, isto é, compreender o processo de envelhecer em sua dinâmica global e específica, associada à presença de patologia crônica. Nesse sentido, a Enfermagem gerontogeriátrica ${ }^{(2-3,11-12)}$ tem importante papel no cuidado e prevenção de agravos à saúde dessas pessoas, configurando-se como indispensável diante das taxas progressivas de mudanças na estrutura etária do país, que se refletem no envelhecimento demográfico ${ }^{(1,12-13)}$.

Diante do exposto, considera-se pertinente compreender as especificidades envolvidas no processo de envelhecimento, estando nesse contexto a capacidade para desenvolver interações sociais-afetivas, numa perspectiva coletiva e singular à pessoa que vivencia uma condição crônica nessa fase da vida. Portanto, delineou-se como objetivo: avaliar as relações sociais e íntimas de pessoas idosas afetadas por hanseníase.

\section{MÉTODO}

Estudo de abordagem quantitativa, realizado em um Centro de Reabilitação em Hanseníase e um hospital público de São Luís, capital do Maranhão - estado do Nordeste brasileiro, considerados referências no atendimento de pessoas afetadas por hanseníase na região(14).

O Centro de Reabilitação em Hanseníase conta com seis consultórios para atendimento e equipe multiprofissional de saúde. Oferece exames de contato, avaliação dermatoneurológica, administração de doses supervisionadas, atividades educativas, treinamento/capacitação em ações básicas e incapacidades, curativos especiais e adaptação de calçados, entre outros. Recebe, diariamente, pacientes com hanseníase oriundos de todo o estado do Maranhão - entre eles, idosos. O hospital, cenário da pesquisa, se configura como hospital geral, oferecendo assistência especializada para pacientes com/sem hanseníase da capital e interior do Maranhão. 
A coleta de dados foi realizada no período de dezembro de 2012 a junho de 2013. Os participantes da pesquisa compreenderam idosos com diagnóstico de hanseníase. Nesse sentido, foi considerada idosa a pessoa com idade igual ou superior a 60 anos, com base na definição da $\mathrm{OMS}^{(4)}$. Como critério de inclusão, adotou-se: idoso, com diagnóstico clínico de hanseníase e em tratamento ambulatorial, que buscou atendimento nos locais de pesquisa no momento da coleta de dados. E como critério de exclusão: existência de problemas psiquiátricos, neurológicos, audiovisuais e de fala, haja vista a possibilidade de comprometerem a fidedignidade das informações emitidas no decurso da coleta de dados. Estes problemas foram identificados a partir dos registros no prontuário.

Para o cálculo da amostra, foi utilizado o StatCalc do Programa Epilnfo, versão 7 do Center for Disease Control and Prevention (CDC) de Atlanta, tendo como base 71 casos de hanseníase em idosos (notificados em 2012 pelas Unidades de Saúde do Município de São Luís - MA, Brasil), frequência esperada de 18,1\%, nível de confiança igual a $95 \%$ e um erro mínimo de 5\%. Após o cálculo, a amostra ficou definida em um número mínimo de 54 casos.

Inicialmente, os idosos foram identificados no livro de registro de casos novos de hanseníase e a partir destas informações, localizou-se o cartão de aprazamento que contém informações sobre o dia do comparecimento do idoso. Nesse dia e, após a consulta de enfermagem, cada idoso foi esclarecido quanto aos objetivos e formas de participação na pesquisa. Foi solicitada a assinatura do Termo de Consentimento Livre e Esclarecido (TCLE) àqueles que aceitaram participar da pesquisa. Após assinatura do TCLE, foi preenchida uma Ficha de Identificação do Idoso e, posteriormente, entregues os questionários World Health Organization Quality of Life - bref (WHOQOL - bref) e World Health Organization Quality of Life - older adults (WHOQOL - OLD), os quais foram preenchidos em um único encontro. Para a aplicação dos questionários, foram obedecidas as instruções do The Whoqo/ Group ${ }^{(15)}$.

O WHOQOL-bref consta de 26 questões, sendo as duas primeiras referentes ao Índice Geral de Qualidade de Vida (IGQV) e, as demais 24 representam cada uma das 24 facetas que compõem o instrumento original, o WHOQOL - 100. Assim, diferente do WHOQOL - 100, em que cada uma das 24 facetas é avaliada a partir de 4 questões, no WHOQOL - bref cada faceta é avaliada por apenas uma questão(15). Essas 24 facetas estão dentro dos seguintes domínios: Físico, Psicológico, Relações Sociais e Meio Ambiente.

O módulo WHOQOL - OLD consiste em 24 questões atribuídas a seis facetas: "Funcionamento do Sensório", "Autonomia", "Atividades Passadas, Presentes e Futuras", "Participação Social", "Morte e Morrer" e "Intimidade". Cada uma das facetas possui 4 questões ${ }^{(16)}$.

Para alcançar o objetivo dessa pesquisa, foram analisados o Domínio das Relações Sociais do instrumento WHOQOL - bref, constituído por três facetas: "Relações pessoais", "Suporte (apoio) social" e "Atividade sexual", bem como a faceta: "Intimidade", do instrumento WHOQOL - OLD.

A análise da Ficha de Identificação do Idoso foi realizada utilizando-se as variáveis: sexo, idade, raça e situação conjugal. O programa utilizado para esse fim foi o Epilnfo, versão 7 do CDC de Atlanta. Tratando-se de estatística descritiva, os resultados foram analisados em números absolutos e percentagem.

As respostas por faceta do WHOQOL - bref e WHOQOL - OLD são obtidas por uma escala do tipo Likertcom cinco pontos (1 a 5). Esses extremos representam $0 \%$ e $100 \%$, respectivamente. Para a análise das respostas na escala de Likert, ocorre a distribuição da frequência, sendo que 1 e 2 representam uma avaliação negativa sinalizando insatisfação, 3 intermediária ou neutra, 4 e 5 uma avaliação positiva, indicando satisfação.

Os domínios são medidos em direção positiva, ou seja, os escores mais altos significam melhor Qualidade de Vida (QV). O escore final obtido em cada domínio e na QV geral pode se transformar em dois tipos de escala: uma de 4 a 20 e outra de 0 a 100. Nesta pesquisa, utilizou-se a escala de 0 a 100, conforme propõe o $\mathrm{WHOQOL}^{(15)}$, por ser facilmente interpretável, dadas as associações com os percentuais.

O Grupo WHOQOL orienta que, para essa análise, deve ser utilizado o programa estatístico Statistical Package of the Social Sciences (SPSS). Ainda que os instrumentos WHOQOL sejam amplamente utilizados e difundidos, a utilização do software SPSS para o cálculo dos resultados desses instrumentos constitui um fator limitantena sua utilização - trata-se de um software de elevado custo e que requer conhecimentos específicos para a sua utilização. Almejando a supressão dessa limitação, um grupo de pesquisadores desdobrou-se em construir ferramentas, a partir do software Microsoft Excel, que foram utilizadas 
nessa pesquisa para a realização do cálculo dos escores e estatística descritiva do WHOQOL bref e do WHOQOL - OLD ${ }^{(17)}$.

A pesquisa foi aprovada pelo Comitê de Ética em Pesquisa (CEP) do Hospital Universitário da Universidade Federal do Maranhão (HUUFMA), sob o título "Qualidade de vida de idosos portadores de hanseníase", em 17 de maio de 2013, pelo parecer $n^{\circ} 289.202$.

Foram levadas em consideração as observâncias éticas que regulamentam a pesquisa em seres humanos em vigor no país, ressaltando-se a garantia de sigilo da identidade na pesquisa, bem como na elaboração do relatório da pesquisa e a ausência de qualquer ônus para o entrevistado. A pesquisa não recebeu financiamento para a sua realização.

\section{RESULTADOS}

Participaram da pesquisa 60 idosos com diagnóstico de hanseníase. Desses, 53,3\% apresentaram idade entre 60 a 69 anos, seguido por $35 \%$ de idosos entre 70 a 79 anos, $10 \%$ entre 80 a 89 anos, e um idoso com idade acima de 90 anos $(1,7 \%)$. Encontrou-se maior frequência do sexo masculino $(58,3 \%)$ em relação ao feminino $(41,7 \%)$. Em relação à raça/cor, parda foi prevalente $(66,6 \%)$; as cores branca e negra foram referidas, respectivamente, por $26,7 \%$ e $6,7 \%$ dos participantes. Em relação à situação conjugal, $45 \%$ dos idosos era casada; alguns relataram que moravam junto com alguém (15\%) ou eram solteiros $(13,3 \%)$, separados $(11,6 \%)$, viúvos $(11,6 \%)$ ou não responderam $(3,5 \%)$.

Analisando-se as facetas do Domínio Relações Sociais do WHOQOL - bref, observou-se que entre os idosos afetados por hanseníase 85\% estavam satisfeitos com suas relações pessoais e $78,3 \%$ consideravam ter grande suporte social. Em se tratando de atividade sexual, $60 \%$ dos idosos estavam satisfeitos, embora seja necessário notar que $40 \%$ estivessem insatisfeitos ou em postura neutra/intermediária.

No que tange às questões da Faceta Intimidade do WHOQOL-OLD, percebeu-se que as opiniões foram divergentes, os idosos se consideravam satisfeitos ou insatisfeitos com relação ao sentimento de companheirismo na vida $(40 \%$ satisfeitos e $38,3 \%$ insatisfeitos), sentimento de amor (45\% satisfeitos e 43,3\% insatisfeitos), oportunidades para amar (35\% satisfeitos e 41,6\% insatisfeitos) e ser amado (36,6\% satisfeitos e $38,3 \%$ insatisfeitos).

Tabela 1 - Respostas dos idosos afetados por hanseníase, segundo as facetas do Domínio Relações Sociais do WHOQOL-bref. São Luís, MA, Brasil, 2013

\begin{tabular}{lcccccc}
\multicolumn{2}{c}{ DOMÍNIO RELAÇÕES SOCIAIS } \\
\cline { 2 - 8 } FACETAS & \multicolumn{1}{l}{ INSATISFAÇÃO } & $\begin{array}{c}\text { POSTURA NEUTRA } \\
\text { OU INTERMEDIÁRIA }\end{array}$ & \multicolumn{2}{c}{ SATISFAÇÃO } \\
\cline { 2 - 8 } & $\mathbf{n}$ & $\%$ & $\mathbf{n}$ & $\mathbf{\%}$ & $\mathbf{n}$ & $\%$ \\
\hline Relações pessoais & 3 & 5 & 6 & 10 & 51 & 85 \\
\hline Suporte (apoio) social & 5 & 8,4 & 8 & 13,3 & 47 & 78,3 \\
\hline Atividade sexual & 12 & 20 & 12 & 20 & 36 & 60
\end{tabular}

Tabela 2 - Respostas dos idosos afetados por hanseníase, segundo as questões da Faceta Intimidade do WHOQOL - OLD. São Luís, MA, Brasil, 2013

\begin{tabular}{|c|c|c|c|c|c|c|}
\hline \multicolumn{7}{|c|}{ INTIMIDADE } \\
\hline \multirow[t]{2}{*}{ QUESTÕES } & \multicolumn{2}{|c|}{ INSATISFAÇÃO } & \multicolumn{2}{|c|}{$\begin{array}{l}\text { POSTURA } \\
\text { NEUTRA OU } \\
\text { INTERMEDIÁRIA }\end{array}$} & \multicolumn{2}{|c|}{ SATISFAÇÃO } \\
\hline & $\mathbf{n}$ & $\%$ & $\mathbf{N}$ & $\%$ & $\mathbf{n}$ & $\%$ \\
\hline $\begin{array}{l}\text { "Até que ponto você tem um sentimento de } \\
\text { companheirismo em sua vida?" }\end{array}$ & 23 & 38,3 & 13 & 21,6 & 24 & 40 \\
\hline "Até que ponto você sente amor em sua vida?" & 26 & 43,3 & 7 & 11,6 & 27 & 45 \\
\hline "Até que ponto você tem oportunidades para amar?" & 25 & 41,6 & 14 & 23,3 & 21 & 35 \\
\hline "Até que ponto você tem oportunidades para ser amado?" & 23 & 38,3 & 15 & 25 & 22 & 36,6 \\
\hline
\end{tabular}




\section{DISCUSSÃO}

Ao ser indagado sobre a satisfação com suas relações pessoais, $85 \%$ relataram que estavam satisfeitos. Sabe-se que as relações familiares e de amizade são as matrizes sociais mais importantes para a pessoa idosa, que auxiliam no enfrentamento das situações diárias e nos sentimentos de solidão, mais perceptíveis na velhice $^{(18)}$, porém, o processo de envelhecimento influencia a dinâmica e funcionalidade familiar, seja em relação às interações sociais ou na sobrecarga de cuidados ${ }^{(6)}$.

Apesar do imaginário constituído acerca da hanseníase, retratada como uma doença que causa desfiguração física e tende a afastar as pessoas, percebe-se que a doença não impede a aproximação e pode também engendrar as relações e a rede social ${ }^{(19)}$. Contudo, em virtude não apenas das modificações no processo de envelhecimento, bem como das conjunturas familiares atuais, é essencial estabelecer uma rede de apoio para os idosos, de modo especial para aqueles que residem sozinhos. Essas redes sociais têm como função promover interações interpessoais, que atuam do apoio emocional ao instrumental, etc ${ }^{(20)}$. A Enfermagem, no gerenciamento dos seus cuidados, deve contemplar a complexidade dessas interações no sentido de identificar potencialidades e limitações para que, assim, possa planejar mecanismos de intervenção.

Ainda sobre a rede de apoio (amigos), os idosos demonstraram-se satisfeitos, embora $21,7 \%$ tenham relatado uma postura neutra ou estivessem insatisfeitos. Depreende-se dessa realidade a importância do convívio social para a manutenção da saúde e prevenção de doenças ou agravos ${ }^{(6-7,13)}$, pois no âmbito da hanseníase, imperam estigmas e se faz premente a ruptura estereotipada de doença incurável ${ }^{(10)}$ que isola o idoso e compromete a sua qualidade de vida. Em estudo desenvolvido na Indonésia com pessoas portadoras de incapacidades relacionadas à hanseníase, 60,3\% tinham problemas de participação na vida social e $35,5 \%$ experimentaram estigma ${ }^{(21)}$. Portanto, a educação formal e em saúde deve se configurar como estratégia resolutiva para a atuação da Enfermagem em meio ao desafio supracitado.

Em relação à satisfação com a vida sexual, $60 \%$ disseram que estavam satisfeitos. Estar satisfeito não quer dizer que possuam ou não vida sexual ativa, pois alguns idosos, mesmo não a tendo, consideram-se satisfeitos com a ausência desse parâmetro de suas vidas. A sexualidade do idoso deve ser compreendida partindo do princípio de que ela se compõe da totalidade deste indivíduo, devendo ser considerado o seu sentido integral. Sendo, portanto, não somente fator biológico, como também biopsicossociocultural (22). A sexualidade quando relacionada ao envelhecimento traduz mitos e tabus, resultando na concepção de que idosos são pessoas "assexuadas"(23), como se o envelhecimento carregasse consigo o desinteresse pela vida e a sexualidade fosse inerente ao jovem.

Em se tratando de hanseníase, há um contexto ainda maior de mitos e tabus, haja vista que no início do século passado, nos debates sanitaristas pelo mundo, o comportamento sexual de pessoas com "lepra" ainda se apresentava como um problema para o planejamento de políticas populacionais, havendo dúvidas recorrentes a respeito do tipo de transmissão da doença, se era transmitida por via sexual ou herdada. Assim, os internos das colônias viviam em pavilhões segregados conforme idade e sexo, e só podiam frequentar os espaços comuns ao mesmo sexo. As histórias eram ainda mais sugestivas quando diziam respeito às pessoas já casadas, nas quais o diagnóstico de hanseníase em um dos pares condenava o casal à separação abrupta ${ }^{(24-26)}$.

Sabe-se que a dinâmica saudável da vida conjugal na velhice está conectada à intimidade, à companhia e à capacidade de expressar sentimentos verdadeiros um para o outro, numa atmosfera de segurança, carinho e reciprocidade $^{(27)}$. Os resultados obtidos na faceta Intimidade revelaram escores mais altos entre os indivíduos que eram casados e moravam juntos, os quais representaram $60 \%$ dos idosos, o que demonstra uma possível mudança na visão atual dessa população que viveu com intensidade o período de hospitais-colônia de hanseníase.

Pôde-se observar que o percentual de idosos que referiram "Satisfação" e "Insatisfação" foi aproximado, quando questionados sobre ter sentimento de companheirismo e de amor em sua vida, ter oportunidades para amar e ser amado por alguém que compartilhe suas intimidades. Essas respostas podem ser explicadas pela presença ou não de companheiro, respectivamente. Em um estudo realizado com idosos com e sem companheiro $^{(27)}$, percebeu-se que há menores escores na intimidade entre aqueles sem companheiro $(66,53 \%)$, em relação aos idosos com companheiro $(73,23 \%)$, embora não tenha sido demonstrada diferença significativa. 
Cumpre salientar, no entanto, que outros fatores, como a falta de informação e o preconceito ao portador de hanseníase, podem agravar o índice de insatisfação em suas relações íntimas. Houve idosos, durante a coleta de dados, que afirmaram ter diminuído consideravelmente ou até mesmo encerrado as relações íntimas com seus cônjuges, alguns por medo de contato físico e íntimo, outros por desconhecimento da forma de transmissão da hanseníase; inclusive, pairando dúvidas se a transmissão poderia se dar por via sexual, por exemplo.

Historicamente as discussões a respeito da sexualidade da pessoa acometida pela hanseníase sempre foram negligenciadas, sendo consideradas pessoas "assexuadas" devido a ser dada maior ênfase à doença ${ }^{(28)}$. Mesmo em obras clássicas como "A história da lepra no Brasil"(29), publicada em meados da década de 50, não há referência direta a sexualidade, namoro e casamento nos hospitais-colônias ${ }^{(24)}$.

Assim, a educação em saúde vem a ser uma estratégia relevante na construção de conceitos que visualizem o idoso como indivíduo livre para vivenciar sua sexualidade, desprendida de mitos e preconceitos que se solidificaram socialmente, sendo necessário considerar que essas ações educativas devem envolver a todos e não somente os idosos, pois o envelhecimento é inerente ao ser humano e questões sobre a sexualidade precisam ser discutidas no percurso de todas as etapas da vida. Portanto, a sexualidade permanece em construção ao longo da trajetória do ser humano, e frente a este processo, destaca-se o papel do enfermeiro como educador, inserindo-o nos espaços de atuação no que se refere à educação sexual ${ }^{(30)}$.

\section{CONCLUSÃO}

As Relações Sociais dos idosos afetados por hanseníase foi um domínio que apresentou alto escore, principalmente nas facetas "Relações Pessoais" e "Suporte (apoio) Social". Tal situação pode ser entendida devido à rede de apoio social na qual a maior parte dos idosos está envolvida. Essas relações pessoais, seja de amizade ou com familiares, trazem expressiva satisfação a essa população, refletindo na sua qualidade de vida.

Diante disso, pensa-se a importância da Enfermagem articular estratégias que contemplem em suas práticas de cuidados à pessoa idosa, em condição crônica de saúde ou não, a interação com redes de apoio. Percebendo, principalmente, que no processo de envelhecimento essas interações podem ser potencialmente prejudicadas pelos estereótipos arraigados culturalmente, com a velhice percebida como condição não natural da vida.

Para a faceta "Atividade Sexual", observou-se menor satisfação dos idosos, bem como na faceta "Intimidade", o que parecer ter relação com a situação conjugal dos mesmos, embora outros fatores, como a falta de informação, tenham provavelmente contribuído para tal resultado. Nessa vertente, a Enfermagem é também de suma importância à realização de ações educativas, com foco na desmistificação da sociedade acerca dos preconceitos associados à hanseníase.

O presente estudo teve como limitação a área e utilização de análise estatística descritiva, dificultando a generalização dos resultados e possíveis inferências, assim como a dificuldade de encontrar trabalhos científicos sobre a temática abordada. Como possibilidades, os instrumentos de coleta se mostraram eficazes para fornecer subsídios para intervenção multidimensional, tanto no âmbito social, como no familiar e íntimo, fatores que devem ser considerados pela Enfermagem no cuidado das pessoas idosas e com hanseníase.

\section{REFERÊNCIAS}

1. Carretta MB, Bettinelli LA, Erdman AL. Reflexões sobre cuidado de enfermagem e a autonomia do ser humano na condição de idoso hospitalizado. Rev. Bras. Enferm. [Internet] 2011; 64(5) [acesso em 04 mar 2015]. Disponível: http://dx.doi.org/10.1590/S003471672011000500024

2. Santos SSC. Concepções teórico-filosóficas sobre envelhecimento, velhice, idoso e enfermagem gerontogeriátrica. Rev. Bras. Enferm. [Internet] 2010; 63(6) [acesso em 05 mar 2015]. Disponível: http:// dx.doi.org/10.1590/S0034-71672010000600025

3. Gonçalves LHT. A complexidade do cuidado na prática cotidiana da enfermagem gerontogeriátrica. Rev. bras. geriatr. gerontol. [Internet] 2010;13(3) [acesso em 08 mar 2015]. Disponível: http://dx.doi.org/10.1590/ S1809-98232010000300016

4. Organização Mundial da Saúde (OMS). Guia global: Cidade amiga do idoso. 2008. [acesso em 08 abr 2015]. Disponível: http://www.who.int/ageing/ GuiaAFCPortuguese.pdf

5. Ministério da Saúde (BR). Envelhecimento e saúde da pessoa idosa. Séria A. Normas e manuais técnicos Cadernos de Atenção Básica n. ${ }^{\circ} 19.2007$. [acesso em 08 
abr 2015]. Disponível: http://bvsms.saude.gov.br/bvs/ publicacoes/abcad19.pdf

6. Nardi EFR, Sawada NO, Santos JLF. Associação entre a incapacidade funcional do idoso e a sobrecarga do cuidador familiar. Rev. Latino-Am Enfermagem [Internet] 2013; 21(5) [acesso em $08 \mathrm{abr}$ 2015]. Disponível: http://dx.doi.org/10.1590/S010411692013000500012

7. Freitas MC, Ferreira MA. Velhice e pessoa idosa: representações sociais de adolescentes escolares. Rev. Latino-Am Enfermagem. [Internet] 2013; 21(3) [acesso em 08 abr 2015]. Disponível: http://dx.doi.org/10.1590/ S0104-11692013000300014

8. Ministério da Saúde (BR). Guia de Vigilância Epidemiológica. Séria A. Normas e manuais técnicos. 2009. [acesso em 04 mar 2015]. Disponível: http:// bvsms.saude.gov.br/bvs/publicacoes/guia_vigilancia_ epidemiologica_7ed.pdf

9. World Health Organization (WHO). Global leprosy situation. Weekly epidemiological record. [Internet]. Geneva: World Health Organization; 2012 [acesso em 04 mar 2015]. Disponível: http://www.who.int/ wer/2013/wer8835.pdf?ua=1

10. Cid RDS, Lima GG, Souza AR, Moura ADA. Percepção de usuários sobre o preconceito da hanseníase. Rev Rene. [Internet] 2012;13(5) [acesso em 08 abr 2015]. Disponível: http://www.revistarene.ufc. br/revista/index.php/revista/article/view/1158

11. Alvarez AM, Reiners AAO, Polaro SHI, Gonçalves LHT, Caldas CP, Unicovsky MAR, et al. Departamento científico de enfermagem gerontológica da associação brasileira de enfermagem. Rev. Bras. Enferm. [Internet] 2013; 66(n.esp) [acesso em 08 abr 2015]. Disponível: http://dx.doi.org/10.1590/S0034-71672013000700023

12. Cruz-Ortiz M, Jenaro-Río C, Pérez-Rodrígues MDC, Hernández-Blanco ML, Flores-Robaina $\mathrm{N}$. Mudanças no contexto do cuidado: desafios para a enfermagem. Rev. Latino-Am Enfermagem [Internet] 2011; 19(4) [acesso em 08 abr 2015]. Disponível: http:// dx.doi.org/10.1590/S0104-11692011000400025

13. Darder JJT, Carvalho ZMF. La interface del cuidado de enfermería com las políticas de atención al anciano. Rev. Bras. Enferm. [Internet] 2012; 65(5) [acesso em 08 abr 2015]. Disponível: http://dx.doi.org/10.1590/S003471672012000500002

14. Secretaria de Estado da Saúde de São Luís (BR). Casos de Hanseníase do Município de São Luís por Faixa Etária em 2012. SINANNET/TABWIN; 2012.

15. The Whoqol Group. Development of the Word Health Organization WHOQOL - bref. Quality of Life. Assesment. Psychol Med. [Internet] 1998; 28(3) [acesso em 08 abr 2015]. Disponível:http://journals.cambridge.
org/action/displayAbstract? fromPage $=$ online $\&$ aid $=257$ 93\&fulltextType=RA\&fileld=S0033291798006667

16. Fleck MPA, Chachamomovich E, Trentini CM. Projeto WHOQOL - OLD: método e resultados de grupos focais no Brasil. Rev Saúde Pública [Internet] 2003; 37(6) [acesso em 08 abr 2015]. Disponível: http:// dx.doi.org/10.1590/S0034-89102003000600016

17. Pedroso B, Pilatti LA, Gutierrez GL, Picinin CT. Cálculo dos escores e estatística descritiva do WHOQOL-bref utilizando o Microsoft Excel. R Bras Qual Vida. [Internet] 2010; 2(1) [acesso em 08 abr 2015]. Disponível: http://dx.doi.org/10.3895/S217508582010000100004

18. Areosa SVC, Benitez LB, Wichmann FMA. Relações familiares e o convívio social entre idosos. Textos \& Contextos [Internet] 2012; 11(1) [acesso em 08 abr 2015]. Disponível: http://revistaseletronicas.pucrs.br/ ojs/index.php/fass/article/view/10495

19. Brakel WH, Sihombing B, Djarir H, Beise $K$, Kusumawardhani L, Yulihane R, et al. Disability in people affected by leprosy: the role of impairment, activity, social participation, stigma and discrimination. Glob Health Action. [Internet] 2012, 5(18394) [acesso em 09 jul 2015]. Disponível: http://dx.doi.org/10.3402/ gha.v5i0.18394

20. Paiva ATG, Bessa MEP, Moraes GLA, Silva MJ, Oliveira RDP, Soares AMG. Avaliação da funcionalidade de famílias com idosos. Cogitare enferm. [Internet] 2011; 16(1) [acesso em 08 abr 2015]. Disponível: http:// dx.doi.org/10.5380/ce.v16i1.21107

21. Cavaliere IAL, Costa SG. Isolamento social, sociabilidades e redes sociais de cuidados. Physis [Internet] 2011; 21(2) [acesso em 09 jul 2015]. Disponível: http://dx.doi.org/10.1590/S0103-73312011000200009.

22. Lyra DGP, Jesus MCP. Compreendendo a vivência da sexualidade do idoso. Nursing. 2007; 9(104):23-30.

23. Coelho DNP, Daher DV, Santana RF, Santo FHE. Percepção de mulheres idosas sobre sexualidade: implicações de gênero e no cuidado de enfermagem. Rev Rene. [Internet] 2010; 11(4) [acesso em 08 abr 2015]. Disponível: http://www.revistarene.ufc.br/vol11n4_ pdf/a18v11n4.pdf

24. Fonseca C. Sexualidade, gênero e afeto nos hospitais-colônias de hanseníase. Cad. Pagu. [Internet] 2013; (41) [acesso em 08 abr 2015]. Disponível: http:// dx.doi.org/10.1590/S0104-83332013000200004

25. Sales JCS, Araujo MPR, Coelho MC, Luz VLES, Silva TCA, Júnior FJGS. Sexualidade de pessoas que vivem com hanseníase: percepção e repercussão. Rev enferm UFPE on line. [Internet] 2013; 7(2) [acesso em 08 abr 2015]. Disponível: http://www.revista.ufpe. $\mathrm{br} / \mathrm{revistaenfermagem/index.php/revista/article/}$ 
26. Mendonça RF. Táticas cotidianas e ação coletiva: a resistência das pessoas atingidas pela hanseníase. Varia Hist. [Internet] 2012; (28)47 [acesso em $10 \mathrm{abr}$ 2015]. Disponível: http://dx.doi.org/10.1590/S010487752012000100016

27. Marchiori GF, Dias FA, Tavares DMS. Qualidade de vida entre idosos com e sem companheiro. Rev enferm UFPE on line. [Internet] 2013; 7(4) [acesso em 08 abr 2015]. Disponível: http://www.revista.ufpe. $\mathrm{br} / \mathrm{revistaenfermagem/index.php/revista/article/}$ view/4053/pdf_2367

28. Santos TMMG, Campelo CL, Costa IA, Rocha SS, Veloso LC. Hanseníase: implicações na sexualidade do portador. Hansen Int. [Internet] 2010; 35(2) [acesso em 08 abr 2015]. Disponível: http://periodicos.ses.sp.bvs. br/pdf/hi/v35n2/v35n2a04.pdf

29. Araújo HS. A história da lepra no Brasil. Rio de Janeiro: Imprensa Nacional; 1946.

30. Alencar DL, Marques APO, Leal MCC, Vieira JCM. Fatores que interferem na sexualidade de idosos: uma revisão integrativa. Ciênc. saúde coletiva [Internet] 2014; 19(8) [acesso em 08 abr 2015]. Disponível: http:// dx.doi.org/10.1590/1413-81232014198.12092013 